\title{
Fabrication of 2.5 Gbps Burst-mode Receiver and its Full Compliance to GPON
}

\author{
Mun Seob Lee*, Byung Tak Lee, Jong Deog Kim, and Dong Soo Lee \\ Optical Communication Research Center, Electronics and Telecommunications Research Institute \\ (ETRI), 1110-6, Oryong-dong, Buk-gu, Gwangju, 500-480, Korea
}

(Received September 25, 2008 : revised October 16, 2008 : accepted October 16, 2008)

\begin{abstract}
In the current GPON market and standard, the line bit rate requirement is changing from 1.25 Gbps to 2.5 Gbps. We fabricate a 2.5 Gbps burst-mode receiver with commercially available blocks and optimize it with an APD bias control. A burst-mode measurement setup is made for the full compliance test with the GPON standard. The device meets the partially defined 2.5 Gbps specs in the current ITU G.984.2 standard, also, supports 1.25 Gbps specs for the coexistence issue in an access network. The full-compliant measurement values can be used as a guideline for fixing "for further study" specs in the current GPON standard at 2.5 Gbps.
\end{abstract}

Keywords: Optical communication, Access networks, PON, Burst-mode receiver.

OCIS codes : (060.0060) Fiber optics and optical communications; (250.3140) Integrated optoelectronic circuits; (060.4250) Networks; (060.4510) Optical communications

\section{INTRODUCTION}

To satisfy the increasing high-bandwidth requirements like IPTV or triple-play services, The passive Optical Network (PON) is being deployed rapidly. PON technology, compared to the other access network technologies, has several merits including low-cost maintenance and easy bandwidth expandability [1]. There are two standard activities for PON, Gigabit capable PON (GPON) by ITU-T [2] [3] and Ethernet PON (EPON) by IEEE [4]. Currently, both have standardized successfully the PON technology at the line bit rate of 1.25 Gbps.

The line bit rate is expected to be upgraded in the near future to expand the PON bandwidth,. The standard spec values have been defined at the line bit rate of both $1.25 \mathrm{Gbps}$ and $2.5 \mathrm{Gbps}$ in the downstream direction. However, for 2.5 Gbps upstream, there are too many "for further study" items in the standard spec values. It is because the upstream transmission is related to the burst-mode operation, which is different from the continuous-mode operation. It is not so easy to fix the spec values without considering commercial availability. At this time, many reports about the 2.5 Gbps burst-mode transmission, especially the receiver side,

*Corresponding author: mslee21@etri.re.kr are required as guidelines for the standardization work. There are few reports [5-7], but they do not show full compliance with the standard.

In this paper, we report a full compliant test about a 2.5 Gbps burst-mode receiver. The receiver module has been fabricated, packaged, and optimized with commercially available blocks and processes. The optimization is mainly performed with a DC bias control of the APD. A burst-mode measurement setup for the full compliance test of the G.984.2 standard is made. The device meets the partially defined 2.5 Gbps specs in the current GPON standard. Also, to address the coexistence issue in the access network, the performance at 1.25 Gbps upstream is measured and discussed. The fullcompliant measurement values can be used as a guideline sfor fixing "for further study" specs in the current GPON standard at 2.5 Gbps.

\section{FABRICATION AND EXPERIMENTAL SETUP}

We fabricate a burst-mode transmitter and a receiver with several commercially available blocks. The burstmode transmitter, located at the optical network terminal (ONT), is made of a burst-mode laser-diode driver 


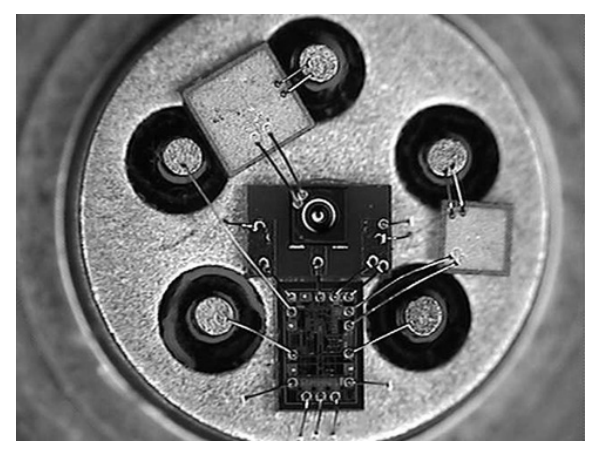

FIG. 1. Top Top view of the packaged TO-CAN.

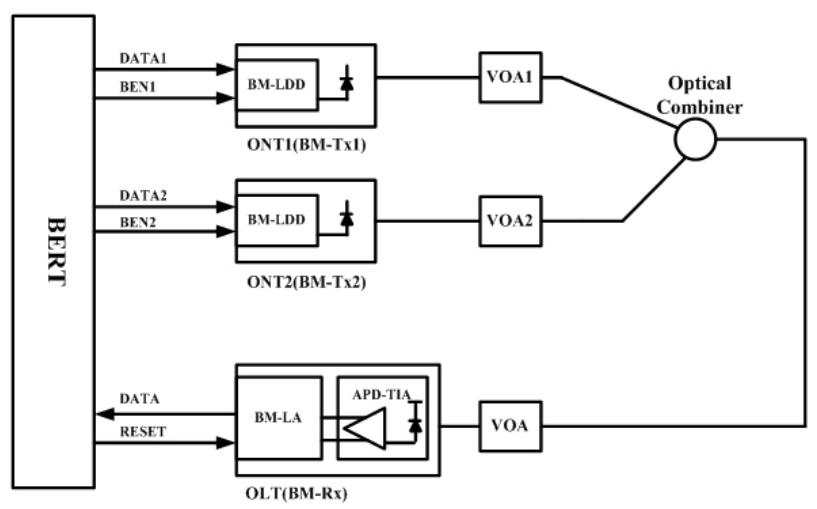

(a)

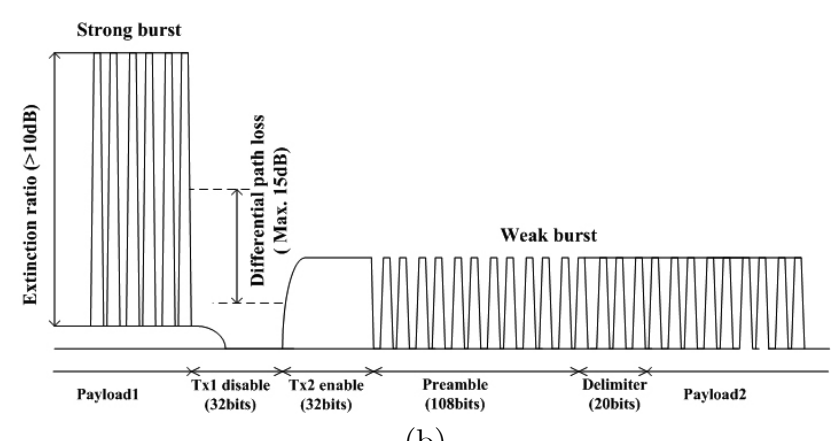

(b)

FIG. 2. Experimental setup for the burst-mode measurement (a) setup (b) burst-mode transmission spec

and a $1310 \mathrm{~nm}$ DFB laser diode. The burst-mode receiver, located at the optical line terminal (OLT), is made of an InGaAs APD, a burst-mode transimpedance amplifier (TIA), and a burst-mode limiting amplifier (LIA). An APD and a TIA are fabricated into a 6-pin TO-CAN package using a bare-chip die-bonding, a wire-bonding, and a mechanical fiber-alignment process. The top view of the packaged TO-CAN is shown in Fig. 1. A DC coupling method between TIA and LIA is used for the rapid recovery of a burst signal and the immunity of the possible 72bit constitutive identification digit (CID).

The experimental setup for a 2.488 Gbps GPON upstream transmission is shown in Fig. 2 (a). The burstmode upstream operation can be decomposed into the

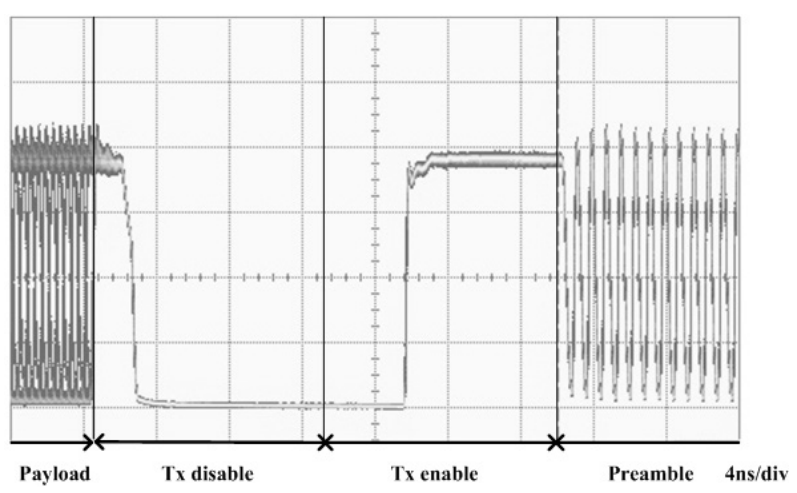

FIG. 3. Burst-mode 2.488 Gbps Tx measurement

burst-mode packet-gap interval and the burst-mode power-level change. As shown in Fig. 2 (b), the worst case at the receiver is that one ONT (including a path loss) transmits the strongest power-level burst signal, followed by a minimum packet-gap interval, and another ONT (including a path loss) transmits the weakest powerlevel burst signal. This situation means the maximum burst-mode power-level change and the minimum burstmode packet-gap interval. The burst-mode power-level change is controlled by two variable optical attenuators (VOA). The maximum burst-mode power-level change, known as differential path loss or soft-and-loud ratio, is recommended to be $15 \mathrm{~dB}$. The burst-mode packetgap interval is emulated by a BERT configuration and synchronized with two burst-mode enable (BEN1 and BEN2) signals from BERT. The minimum burst-mode packet-gap interval, i.e. $\mathrm{T} \times 1$ disable plus $\mathrm{T} \times 2$ enable, is recommended to be 64 bit-time (=32 bit-time +32 bit-time).

As the burst mode transmitters, ONT1 has the output power of $2.37 \mathrm{dBm}$ and $\mathrm{ONT} 2,2.51 \mathrm{dBm}$ at $1310 \mathrm{~nm}$. The basic characteristics in the optical signal domain are measured as shown in Fig. 2. The extinction ratio is measured to be $12.53 \mathrm{~dB}$, which shows enough margin compared to the G.984.2 spec value of $10 \mathrm{~dB}$. The rising and falling time, measured from $10 \%$ to $90 \%$, are $104 \mathrm{psec}$ and 202psec, respectively. The launched power without any digital signal input to the laser diode, of which the level is required to be low enough to avoid signal interferences, is measured to be $-62 \mathrm{dBm}$. The burst-mode enable and disable time in the optical signal domain is measured to be $6.65 \mathrm{~ns}$ and $2.15 \mathrm{~ns}$ which is below 32 bit-time at 2.488 Gbps, respectively, as shown in Fig. 3. Digitally automatic power control circuitry is used for the fast response time and keeping a constant output power within a packet time.

\section{RESULTS AND DISCUSSION}

To emulate the maximum burst-mode power-level 


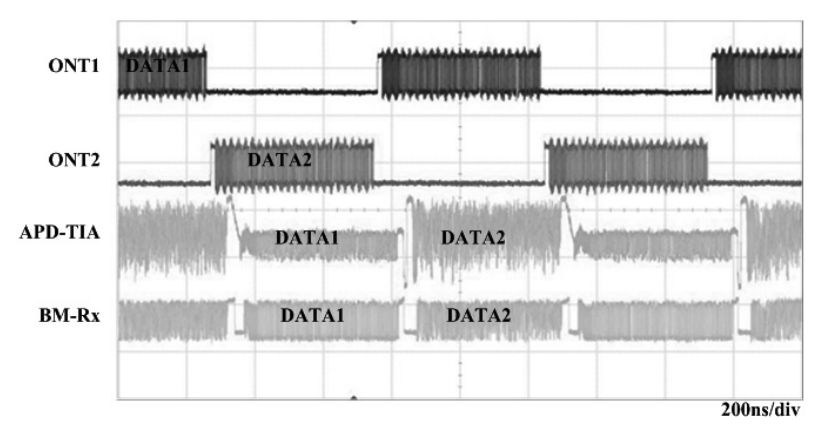

FIG. 4. Burst-mode Rx measurement (ONT1, ONT2: digital signal waveform fed into each ONT, APD-TIA: signal waveform right after TIA with ONT1 output power of $-22.6 \mathrm{dBm}$ and ONT2 output power of -7.6 $\mathrm{dBm}, \mathrm{BM}-\mathrm{Rx}$ : signal waveform right after LIA)

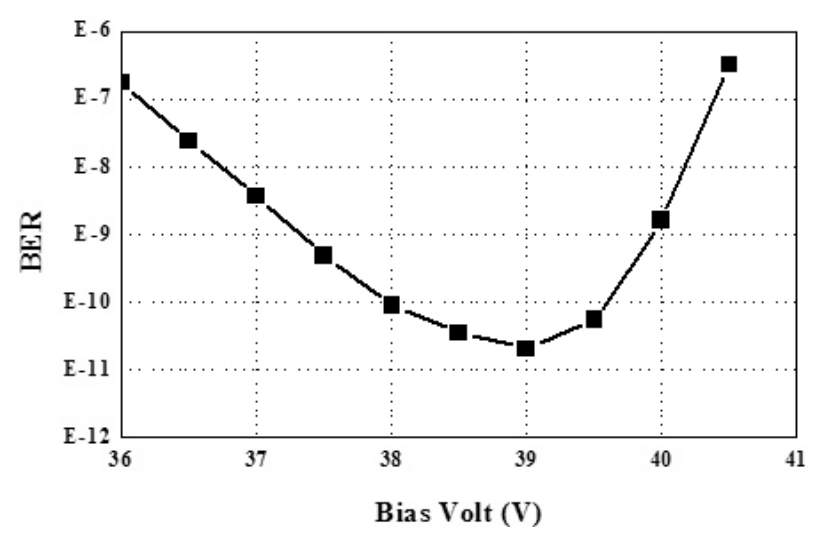

FIG. 5. BER performance versus reverse bias voltage of APD

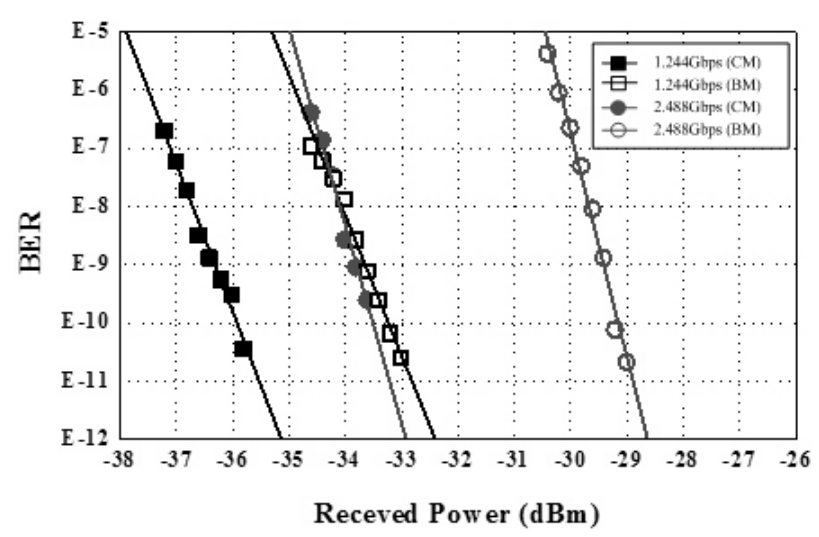

FIG. 6. BER performance (CM: continuous-mode operation, BM: burst-mode operation)

change between a strong burst and a weak burst, we control two VOAs in Fig. 2(a) to set the output power of ONT1 and ONT2 to be $-22.6 \mathrm{dBm}$ and $-7.6 \mathrm{dBm}$, repectively. The difference value is the same with the differential path loss spec, $15 \mathrm{~dB}$, in G.984.2. Also, the RESET signal from BERT in Fig. 2(a) is controlled to be positioned within the Tx2 enable signal interval. In a real system, this RESET signal will be generated from the layer-2 chip like MAC (Media Access Contol). Its role is to initialize the gain-mode and the thresholdlevel for the TIA and LIA circuitry, respectively, and help these circuitries rapidly adapt from a burst to a burst within the narrow time interval. It improves the BER performance when it is properly positioned. In the G.984.2 standard, maximum 140 bits (=Tx enable 32 bits + preamble 108 bits) at $2.488 \mathrm{Gbps}$ is given for this adaptation time interval.

The burst-mode Rx waveform is shown in Fig. 4. Each digital signal (=all-1-padding 32bits + preamble 108 bits + delimiter 20 bits + payload + all-0-padding 32 bit) from BERT is fed into ONT1 and ONT2. Each digital signal is synchronized with a BEN signal, which turns on the burst-mode laser diode. The signal waveform right after the TIA is shown in Fig. 4, where the burstmode power-level change is compressed due to the automatic gain control behavior of the TIA. The signal waveform right after the LIA is fed into BERT to measure the BER performance.

The bias control of the APD can enhance the burstmode BER performance. We show that there is an optimum bias point to make the best performance in Fig. 5. For the APD, there is a multiplication factor, $\mathrm{M}$, which is proportional to the bias voltage below the breakdown voltage. When the bias voltage is relatively low and increasing, the SNR value is enhanced because the signal is amplified but the noise is nearly fixed (thermal-noise limited region). However, when the bias voltage is too large, the SNR value is degraded because the noise increases more rapidly than the amplified signal (short-noise limited region) [8]. We measure the optimum value to be -39 volts $\mathrm{DC}$ bias with the optical power of $-29.0 \mathrm{dBm}$. The following measurement is performed with this fixed DC bias value for the optimum performance.

The burst-mode (BM) BER performance is measured with the line bit rates of $1.244 \mathrm{Gbps}$ and $2.488 \mathrm{Gbps}$, and is shown in Fig. 6. Also, the continuous-mode (CM) BER performance is measured for comparison. A PRBS pattern with $223-1$ is used. The optical penalty due to the burst-mode effects is measured to be $4.2 \mathrm{~dB}$ at $2.488 \mathrm{Gbps}, 2.4 \mathrm{~dB}$ at $1.244 \mathrm{Gbps}$. The main reason is reported as the uncertainty of the decision threshold value, which is influenced by the dc offset value, the extinction ratio, the sample-and-hold filter bandwidth, the preamble length, the gain of the APD, and the softand-loud ratio [9].

Our results are summarized in Table 1 . The burst-mode receiver meets the partially defined 2.5 Gbps specs in the G.984.2 standard. And, its main performance, which values are corresponding to "for further study" items in the standard, shows a sensitivity of $-29.1 \mathrm{dBm}$ with 10-10 BER and a wide dynamic range of $21.5 \mathrm{~dB}$ (= receiver overload - receiver sensitivity) at 2.488 Gbps. We think that this performance is enough for the real 
TABLE 1. Results summary of burst-mode upstream transmission and its comparison to G.984.2 (FFS: "for further study")

\begin{tabular}{|c|c|c|c|c|c|}
\hline Parameter & Unit & $\begin{array}{c}\text { G.984.2 } \\
\text { at } 2.488 \mathrm{Gbps}\end{array}$ & $\begin{array}{c}\text { Our } \\
\text { measure-ment }\end{array}$ & $\begin{array}{c}\text { G.984.2 } \\
\text { at } 1.244 \text { Gbps }\end{array}$ & $\begin{array}{c}\text { Our } \\
\text { measure-ment }\end{array}$ \\
\hline Nominal bit rate & bps & 2.488 & 2.488 & 1.244 & 1.244 \\
\hline Optical budgets & $\mathrm{dB}$ & 29 & 29 & 29 & 29 \\
\hline Differential optical path loss & $\mathrm{dB}$ & 15 & 15 & 15 & 15 \\
\hline Burst mode overhead & bits & 192 & 192 & 96 & 96 \\
\hline Guard time & bits & 64 & 64 & 32 & 32 \\
\hline Min. launched power & $\mathrm{dBm}$ & $F F S$ & 2.4 & 1.5 & 2.4 \\
\hline $\begin{array}{l}\text { Launched power } \\
\text { without input to the transmitter }\end{array}$ & $\mathrm{dBm}$ & $F F S$ & -60.0 & -37.0 & -60.0 \\
\hline Extinction ratio & $\mathrm{dB}$ & $F F S$ & 12.5 & 10 & 16.3 \\
\hline CID & bits & $F F S$ & 72 & 72 & 72 \\
\hline Receiver sensitivity & $\mathrm{dBm}$ & $F F S$ & -29.1 & -27 & -33.5 \\
\hline Receiver overload & $\mathrm{dBm}$ & $F F S$ & -7.6 & -8.0 & -7.6 \\
\hline
\end{tabular}

access network deployment when compared to the performance of the sensitivity of $-33.5 \mathrm{dBm}$ and a dynamic range of $25.9 \mathrm{~dB}$ at 1.244 Gbps. In addition, we can confirm that its performance at $1.244 \mathrm{Gbps}$ is within the defined specs of the G.984.2 standard, which is important in the coexistence issue in an access network. The transmission penalty over $20 \mathrm{~km}$ is measured to be $0.9 \mathrm{~dB}$ at $2.488 \mathrm{Gbps}$. The burst-mode receiver is fabricated with several commercially available blocks and processes, so, these full-compliant measurement values can be used as a useful guideline for fixing remained specs in the current GPON standard at 2.5 Gbps.

\section{CONCLUSION}

We fabricate a burst-mode transmitter and a burstmode receiver at $2.488 \mathrm{Gbps}$ with several commercially available blocks and processes. A burst-mode measurement setup is made for the full compliance test with the ITU-T G.984.2 standard. The burst-mode receiver is optimized by an APD bias control and its burstmode performance is measured at 1.244 Gbps and 2.488 Gbps line bit rate, respectively. All measurement values satisfy the partially defined standard spec values at 2.488 Gbps and the all spec values at 1.244 Gbps. Also, performance items corresponding to "for further study" specs at 2.488 Gbps are measured including the sensitivity of $-29.1 \mathrm{dBm}$ and the overload of $-7.6 \mathrm{dBm}$. We think that these full-compliant measurement values can be used as a useful guideline for fixing remained specs in the current GPON standard at 2.5 Gbps.

\section{REFERENCES}

[1] F. Effenberger, D. Cleary, O. Haran, G. Kramer, R. D. Li, M. Oron, and T. Pfeiffer, "An Introduction to PON Technologies," IEEE. Communications Magazine, vol. 45, no. 3, pp. S17-S25, 2007.

[2] Gigabit-capable passive optical networks (GPON): General characteristics, ITU-T Recommendation G.984.2, 2003.

[3] ITU-T Rec. G. 984.2. Amendment 1, "New Appendix III - Industry best practice for $2.488 \mathrm{Gbit} / \mathrm{s}$ downstream, 1.244 Gbit/s upstream,” Feb, 2006.

[4] IEEE 802.3ah Ethernet in the first Mile Task Force, D3.3, April, 2004.

[5] C. Yu and D. Li "A 2.5 Gb/s CMOS Burst-Mode Limiting Amplifier for GPON System,” IEEE International Symposium on Circuits and System, New Orleans, pp. 25382541, May, 2007.

[6] E. Hugues-Salas, R. Razavi, T. Quinlan, M. Thakur, and S. Walker, "A $2.5 \mathrm{~Gb} / \mathrm{s}$ Edge-Detecting Burst-Mode Receiver for GPON Access Networks," Optical Fiber Communication and the National Fiber Optic Engineers Conference, Anaheim, pp.1-3, March, 2007.

[7] W. Z. Chen and R. M. Gan, "A Single Chip 2.5 Gbps CMOS Burst Mode Optical Receiver,” Proc. IEEE Symp. VLSI Circuits, Hawaii, pp.120-121, 2006.

[8] G. P. Agrawal, Fiber-Optic Communication Systems 2nd ed. (John Wiley \& Sons, USA, 1997) Chapter 4.

[9] P. Ossieur, X. Z. Qiu, J. Bauwelinck, and J. Vandewege, "Sensitivity penalty calculation for burst-mode receivers using avalanche photodiodes," IEEE J. Lightwave Tech., vol. 21, no. 11, pp. 2565 - 2575, 2003. 